University of New Hampshire

University of New Hampshire Scholars' Repository

Space Science Center

Institute for the Study of Earth, Oceans, and

Space (EOS)

10-1997

\title{
A prototype for SONTRAC, a scintillating plastic fiber tracking detector for neutron imaging and spectroscopy
}

James M. Ryan

University of New Hampshire, James.Ryan@unh.edu

John R. Macri

University of New Hampshire - Main Campus, John.Macri@unh.edu

Mark L. McConnell

University of New Hampshire - Main Campus, mark.mcconnell@unh.edu

C B. Wunderer

University of New Hampshire - Main Campus

D Holslin

Science Applications International Corporation

See next page for additional authors

Follow this and additional works at: https://scholars.unh.edu/ssc

Part of the Astrophysics and Astronomy Commons

\section{Recommended Citation}

Ryan, J.M.; Macri, J.R.; McConnell, M.L.; Wunderer, C.B.; Holslin, D.; Polichar, A.; Baltgalvis, J., "A prototype for SONTRAC, a scintillating plastic fiber tracking detector for neutron imaging and spectroscopy," Nuclear Science Symposium, 1997. IEEE , vol., no., pp.896,900 vol.1, 9-15 Nov 1997.

This Conference Proceeding is brought to you for free and open access by the Institute for the Study of Earth, Oceans, and Space (EOS) at University of New Hampshire Scholars' Repository. It has been accepted for inclusion in Space Science Center by an authorized administrator of University of New Hampshire Scholars' Repository. For more information, please contact Scholarly.Communication@unh.edu. 


\section{Authors}

James M. Ryan, John R. Macri, Mark L. McConnell, C B. Wunderer, D Holslin, Aaron R. Polichar, and Janis Baltgalvis 


\title{
A Prototype for SONTRAC, a Scintillating Plastic Fiber Tracking Detector for Neutron Imaging and Spectroscopy
}

\author{
James M. Ryan, John R. Macri, Mark L. McConnell, Cornelia B. Wunderer \\ Space Science Center \\ University of New Hampshire \\ Durham, NH 03824
}

\author{
Daniel Holslin, Aaron Polichar, Janis Baltgalvis \\ Science Applications International Corporation \\ San Diego, CA 92121
}

\begin{abstract}
We report on tests of a prototype detector system designed to perform imaging and spectroscopy on 20 to 250 MeV neutrons. Although developed for the study of high-energy solar flare processes, the detection techniques employed for SONTRAC, the SOlar Neutron TRACking experiment, can be applied to measurements in a variety of disciplines including atmospheric physics, radiation therapy and nuclear materials monitoring.

The SONTRAC instrument measures the energy and direction of neutrons by detecting double neutron-proton scatters and recording images of the ionization tracks of the recoil protons in a densely packed bundle of scintillating plastic fibers stacked in orthogonal layers. By tracking the recoil protons from individual neutrons, the kinematics of the scatter are determined. This directional information results in a high signal to noise measurement. SONTRAC is also capable of detecting and measuring high-energy gamma rays $>20 \mathrm{MeV}$ as a "solid-state spark chamber." The self-triggering and track imaging features of a prototype for tracking in two dimensions are demonstrated in calibrations with cosmic-ray muons, 14 to $\sim 65 \mathrm{MeV}$ neutrons and $\sim 20 \mathrm{MeV}$ protons.
\end{abstract}

\section{INTRODUCTION AND MOTIVATION}

Neutrons above the nuclear binding energy are ubiquitous in cosmic ray interactions, whether those reactions occur on the surface of the Sun or in the earth's atmosphere. They are notoriously difficult to measure because they lack charge. Consequently, they do not interact readily with detector material. Directional neutron telescopes based on double scatters have been used for many years. [1], [2], [3], [4]. By using a neutron telescope rather than omnidirectional spectrometers, we are able to reject most of the background due to geometrical considerations, thereby greatly enhancing the signal-to-noise ratio.

Double scatter tracking detectors such as described here can be very effective in high background environments and have the potential to perform measurements of unprecedented quality. Such measurements will have applications in a broad range of disciplines including solar physics, atmospheric physics, radiation therapy and nuclear materials monitoring.

When high-energy charged particle reactions occur on the surface of the Sun, neutrons carry away information about the spectrum of ions that produced them. They can be used as diagnostic measures of the accelerated ion spectrum in solar flares [5], [6], [7], [8]. In the earth's atmosphere neutrons above the nuclear binding energy produce so-called soft error upsets (SEUs) in microcircuitry and they represent a radiation hazard for personnel at high altitudes [9], [10], [11], [12], [13], [14], [15].

Proton radiotherapy (PRT) is a technologically advanced means of treating cancer and other diseases. A high energy proton beam can accurately deliver a precise radiation dose to the lesion while minimizing the dose to the surrounding tissue. The success of PRT is based on the precision with which the dose is deposited in the tumor volume [16]. Neutron tracking detectors can also be employed to accurately locate nuclear materials (waste, spills). Unlike moderated ${ }^{3} \mathrm{He}$ counters, these detectors would be much lighter as well as directionally sensitive.

\section{TRACKING DETECTOR CONCEPT}

We have been investigating a tracking detector concept for measuring neutrons in the 20 to $250 \mathrm{MeV}$ range. It employs a closely packed bundle of square cross section plastic-scintillator fibers. Fig. 1 represents a $3 \mathrm{~mm}$ cubic segment of a much larger bundle. The fibers are arranged in stacked planes with the fibers in each plane orthogonal to those in the planes above and below. This alternating orientation allows one to record stereoscopic images and track 


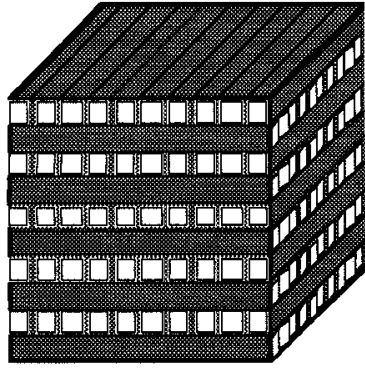

Fig. 1. Segment (3 mm cube) of a larger fiber bundle,

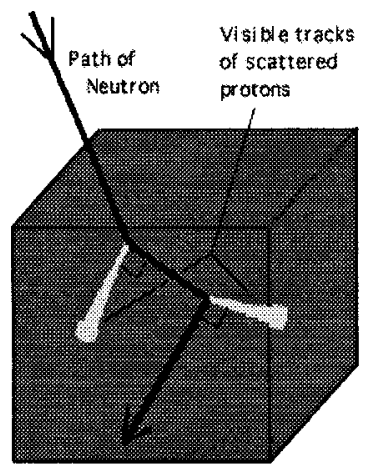

Fig. 2. N-P kinematics. ionizing particles in three dimensions in the scintillating fiber block.

The tracking detector measures the energy and direction of incident neutrons by imaging the ionization tracks of recoil protons. The nonrelativistic double-scattering schematic in a solid block of plastic scintillator is shown in Fig. 2. Neutrons undergo elastic scattering off hydrogen within the organic plastic-scintillator fibers, scattering at right angles with respect to the scattered proton at non-relativistic energies. The Bragg peak, resulting from greater ionization near the end of the track, is used to determine proton track direction. A second proton scatter of the scattered neutron provides spatial information that is necessary and sufficient to determine the incident neutron energy and direction. With sufficient event statistics, an image and spectrum of the neutron source can be constructed. The angular and energy resolution are dependent upon the ability to precisely track the recoil protons and measure the scintillation light.

A functional diagram of an experiment utilizing the SONTRAC concept is shown in Fig. 3. This serves to illustrate the major components which would be required in any realistic system. The detector's spectroscopic, track detection and imaging components cover the entire light emitting area of the scintillating fiber bundle and are duplicated in the orthogonal dimension (not shown). The scintillation light signal is collected and processed at both ends of the fiber bundle. At one end a signal above threshold from a photomultiplier tube (PMT) fires a discriminator that in turn provides a signal to the trigger logic circuitry. At the other end of the fiber bundle, fiber-optic tapers and a pair of image intensifiers demagnify, capture and hold the scintillation-light image of the ionization track(s) for readout by the CCD camera. The first image intensifier in this chain is always ON. Its phosphor holds the image for approximately $1 \mathrm{~ms}$. The second image intensifier in this chain is normally in the gated-OFF condition and no image signal is passed to the CCD sensor. However, when the trigger logic registers the proper coincidence, the track image and PMT pulse height data are acquired and passed to an event

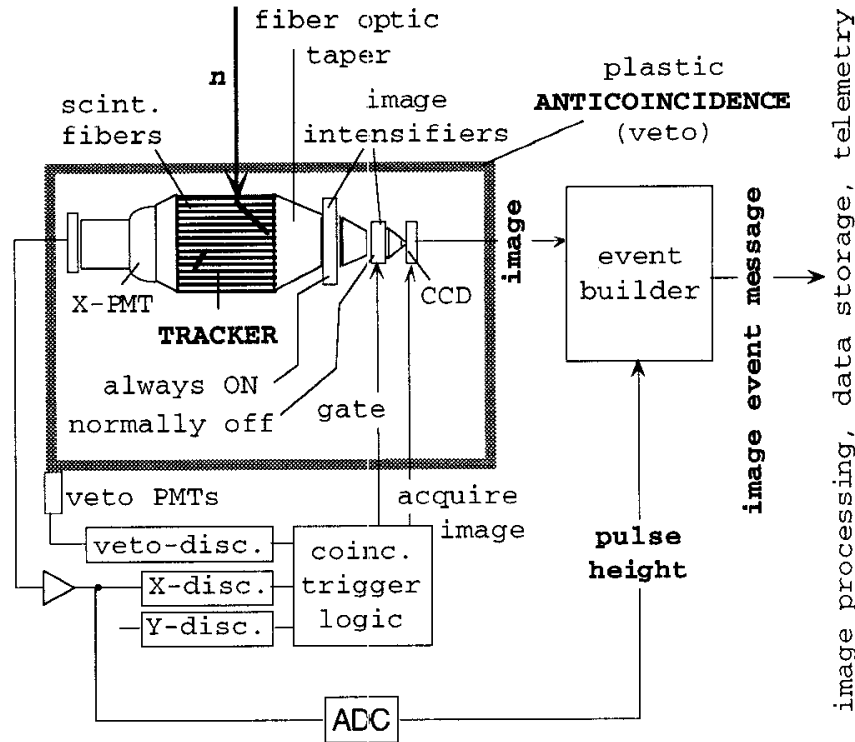

Fig. 3. Functional diagram of the tracker signal processing logic and the major components of a complete instrument system.

builder and combined with auxiliary information for subsequent event-by-event analysis.

A $4 \pi$ anticoincidence (veto) shield composed of plastic scintillator surrounds the tracker. While transparent to neutrons and gamma rays, a charged particle cannot enter the detector volume without generating a trigger pulse. Events coinciding with this trigger pulse are rejected (vetoed). Similarly, charged particles generated by interactions in the detectors and escaping the detector volume will also be detected. These escaping particles represent lost energy and we can choose to accept or reject such events.

The fundamental instrument design was studied extensively with Monte Carlo simulations [17], [18], [19]. It suffered at the time from a lack of technology and existed in simulations only. This technology has been applied to high energy physics experiments [20], and has since become available at a reasonable price. We have assembled and tested a small laboratory prototype which is described below.

\section{Prototype Description AND Test SETUP}

The tracker prototype was developed to demonstrate the tracking capabilities and to address fundamental science and engineering issues related to the calibration and design of space flight instrumentation.

Fig. 4 is a photograph of the prototype tracker. Fig. 5 is a schematic representation of its major components and the setup. It was assembled by SAIC from commercially available parts that can be replaced or interchanged in performance tradeoff studies [21]. To save cost it is small and limited to tracking in two dimensions. Larger scale, threedimension tracking prototypes will follow when optimal 


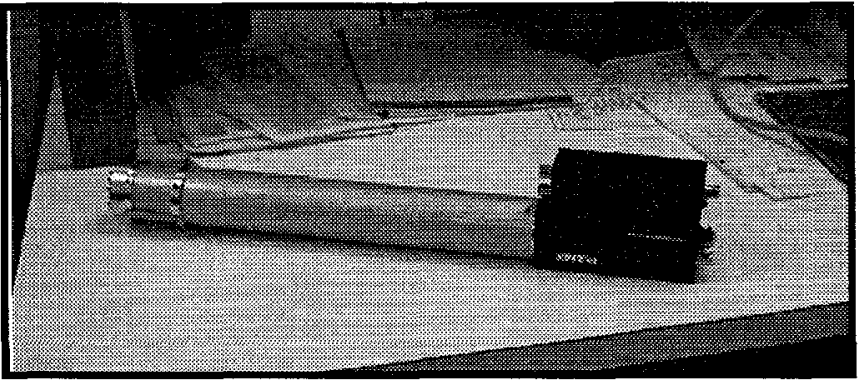

Fig. 4. The SONTRAC prototype detector. The Pulnix camera (TM-9701) is shown to the right and the PMT to the left.

parameters such as phosphor type(s) for the image intensifiers are determined.

The prototype has a $10 \mathrm{~cm}$ long bundle of $250 \mu \mathrm{m}$ square (230 $\mu \mathrm{m}$ active) multiclad organic scintillating plastic fibers (Bicron BCF-99-55) within a $12.7 \mathrm{~mm}$ square envelope. The thickness of the scintillating fibers was chosen such that a 10 $\mathrm{MeV}$ recoil proton traverses several fibers before stopping. The fiber pitch is $300 \mu \mathrm{m}$ (including cladding and EMA) and the calculated range of a $10 \mathrm{MeV}$ proton ( $50 \%$ of the proposed neutron threshold energy) is $1.25 \mathrm{~mm}$, equivalent to $\sim 4$ fibers. The PMT is a bialkali photocathode device from Thorn EMI. Two $18 \mathrm{~mm}$ diameter single MCP generation-2 image intensifiers from DEP are employed. The S20 photocathode for the first image intensifier was selected for optimum response to the scintillation light signal. The P43 phosphor will hold the image for approximately $1 \mathrm{~ms}$. The second intensifier's photocathode (S25) and phosphor (P43) were selected to provide good spectral matches to the output of the first intensifier and the input to the CCD sensor respectively. The CCD camera (Pulnix TM-9701) is an inexpensive, progressive scanning camera with digital readout and control, asynchronous external trigger and full frame shutter capability. The frame grabber and image processor are

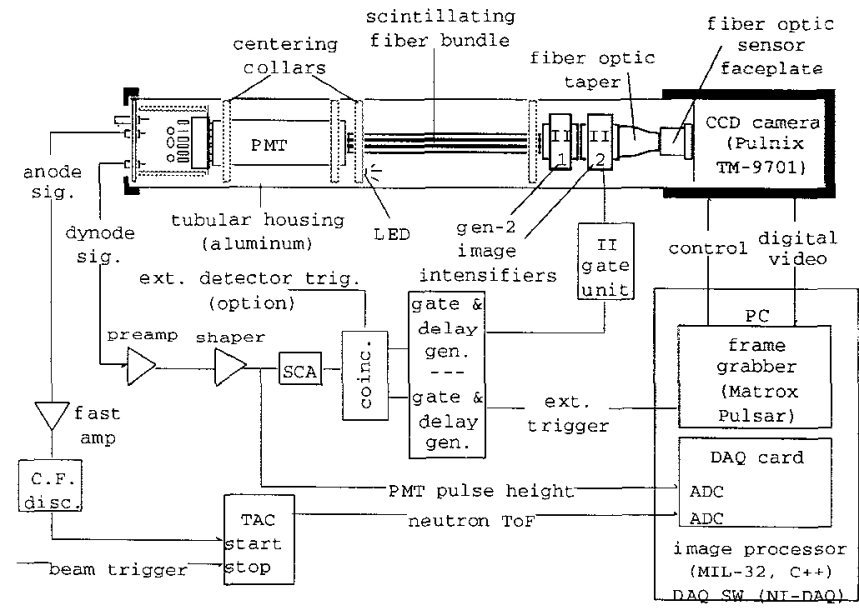

Fig. 5. Schematic illustrating the components of the SONTRAC prototype and the measurement setup. from Matrox and operate in a Pentium PC. The logic and PMT signal amplifier circuitry consists of NIM-standard laboratory modules. A data acquisition (DAQ) card and supporting software in the PC are used to measure PMT pulse height and neutron time-of-flight (ToF) and to merge this information with each recorded image. The light from an internally mounted, externally driven LED is seen by both the PMT and the imaging electronics. Measurements with the LED are used to help establish appropriate gains, gate delays and integration times and to map the scintillating fiber boundaries onto the $\mathrm{CCD}$ pixel matrix.

\section{Prototype PeRformance}

Our ability to track recoil protons has been demonstrated in the lab. The SONTRAC prototype was exposed to 14 $\mathrm{MeV}$ neutrons at San Diego State University (May, 1997). The track of a recoil proton near our trigger threshold energy is shown in Fig. 6. On the left is a portion of the CCD camera image of the track and on the right are the same data averaged over the corresponding fibers. The dynamic range of the fiber brightness expected from an $11 \mathrm{MeV}$ proton is $\sim 2$. This agrees well with the density distribution in the figure. The bright fiber to the right of the proton track in Fig. 6 is probably a related second scatter. Near threshold energies and with tracking in only two dimensions little additional information is available.

More recently (September, 1997) the SONTRAC prototype was exposed to $20 \mathrm{MeV}$ and $27 \mathrm{MeV}$ neutrons and to $\sim 20 \mathrm{MeV}$ protons at the Crocker Laboratory cyclotron facility at the University of California at Davis. Additional measurements were made with $\sim 65 \mathrm{MeV}$ neutrons, although it was not possible to get a clean beam in the time available. Fig. 7 shows two CCD images of neutron interactions in the prototype fiber bundle. On the top is a double scatter event displaying two recoil proton tracks from a neutron $(-65 \mathrm{MeV})$ incident from the top of the figure. On the bottom is a single proton recoil track from a $27 \mathrm{MeV}$ neutron incident from the top left of the figure. The calibration mask of the entire fiber bundle is superposed on the track image. Note that the Bragg
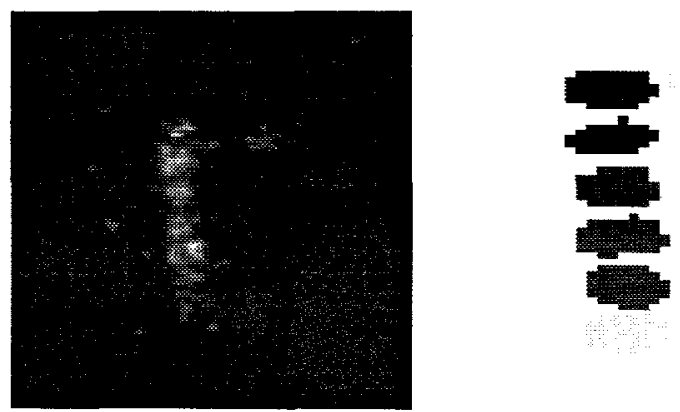

Fig. 6. A $\sim 11 \mathrm{MeV}$ proton track image. Neutron incidence is from the bottom. 

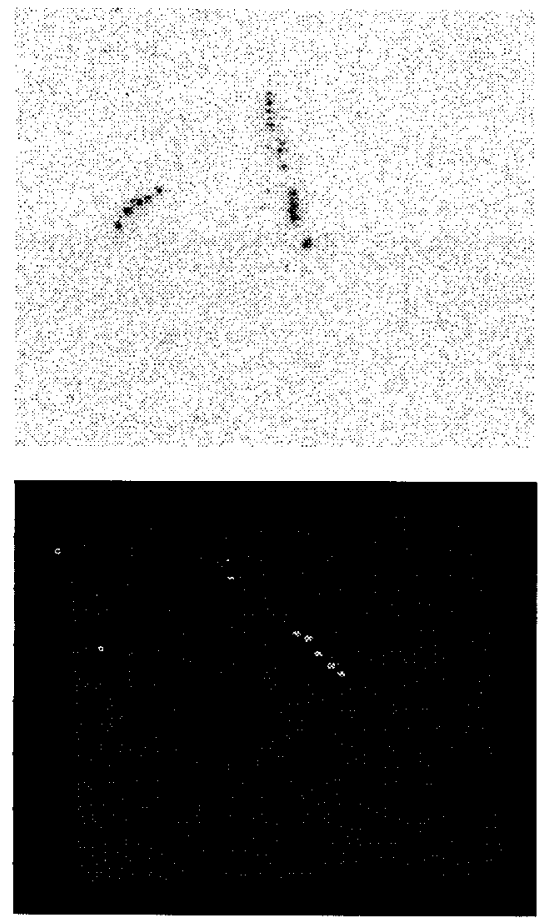

Fig. 7. Raw CCD images of neutron interactions. Top: double scatter from $\sim 65 \mathrm{MeV}$ neutron. Bottom: Proton recoil track from $27 \mathrm{MeV}$ neutron.

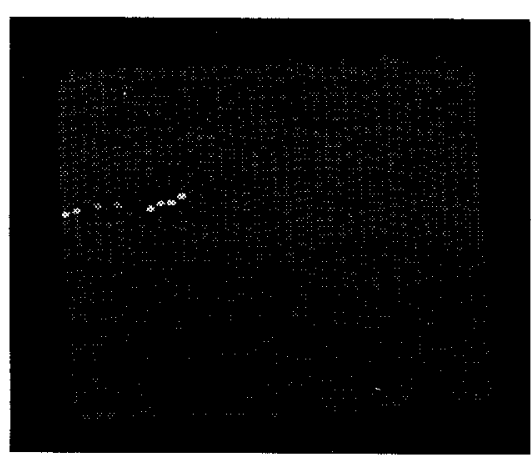

Fig. 8. Track of $\sim 20 \mathrm{MeV}$ proton incident from left.

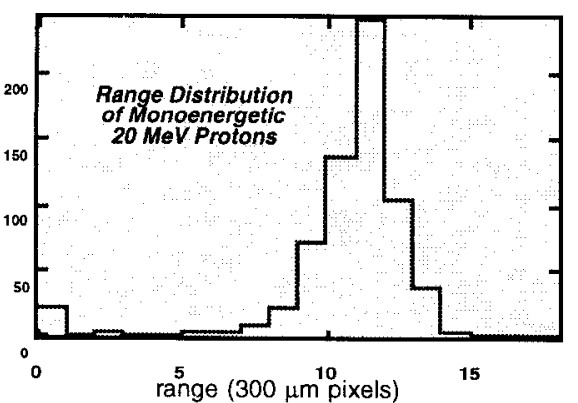

Fig. 9. Histogram of track lengths for monoenergetic protons. peak effect, greater ionization at the end of the track, is evident thus permitting determination of track direction. "Holes" in the track images are due to passage of the ionizing particles through the passive cladding and EMA materials.

Fig. 8 shows the track of a $\sim 20 \mathrm{MeV}$ proton incident from the left of the figure. Again the calibration mask of the fiber bundle is superposed on the track image. In Fig. 8 the CCD pixel intensity was averaged over individual fibers. Note again the evidence of track direction and that the track of the incident proton (Fig. 8) starts at the edge of the bundle. Fig. 9 is a histogram of the track lengths measured for $650 \sim 20$ $\mathrm{MeV} \quad$ protons incident normal to the fiber bundle surface.

Fig. 10 is a cosmic-ray muon track image recorded with the SONTRAC prototype. The track traverses the full $12.7 \mathrm{~mm}$ width of the fiber bundle and demonstrates the detector's ability to track

minimum ionizing radiation. As such it can serve to track conversion electrons in high energy ( $>20 \mathrm{MeV}$ ) gamma detectors. We calculate that $\sim 4$ photoelectrons/fiber are generated for amplification within the image intensifier.

\section{PROPOSED INSTRUMENTATION}

We have proposed to NASA for the development of scintillating fiber tracking instrumentation to measure high energy solar flare emission during the upcoming solar maximum. A conceptual sketch of a larger volume detector for particle tracking in three dimensions is shown in Fig. 11 . It employs orthogonal layers of scintillating fibers in a $10 \times 10 \times 10 \mathrm{~cm}$ bundle and an orthogonal pair of event detection and track imaging systems each having one PMT (left)and one image intensifier /

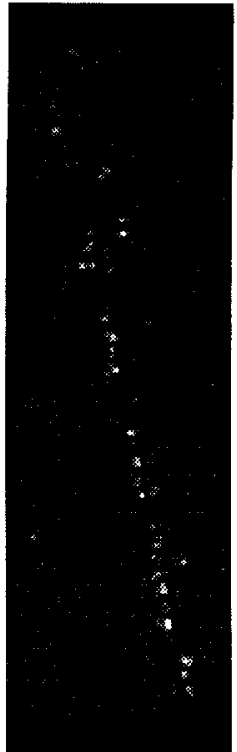

Fig. 10. A cosmic-ray muon track CCD chain (right)

The fiber block will be surrounded by charged particle detectors to (1) reject cosmic ray protons and electrons and (2) to detect the escape of secondary charged particles from reactions within the detector. The flight instrumentation would occupy a space with dimensions less than $50 \times 50 \times$ $20 \mathrm{~cm}$ and have a total mass less than $26 \mathrm{~kg}$. We estimate that the proposed instrumentation will have an effective area for detecting and measuring 15 to $60 \mathrm{MeV}$ neutrons of 2 $\mathrm{cm}^{2}$. This is approximately the neutron effective area of the imaging Compton telescope, COMPTEL, a $1460 \mathrm{~kg}$ instrument.

The typical energy resolution is on the order of $10 \%$ or better for the majority of neutron events. Angular resolution is largely determined by the pitch of the fibers, i.e., the uncertainty in the end points of the particle tracks. For a $45^{\circ}$ scatter and $300 \mu \mathrm{m}$ fiber pitch it ranges

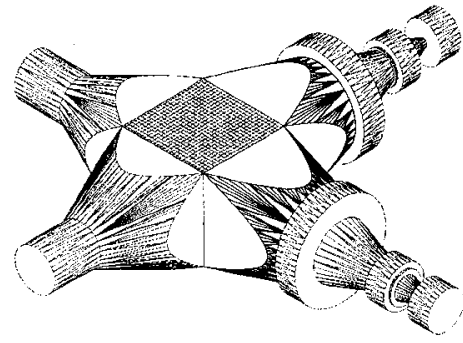

Fig. 11. Conceptual sketch of a 10 $\times 10 \times 10 \mathrm{~cm}$ scintillating plastic fiber tracking detector.

from $23^{\circ}$ at $20 \mathrm{MeV}$ to

$5^{\circ}$ at $50 \mathrm{MeV}$ to $0.7^{\circ}$ at $200 \mathrm{MeV}$. This angular resolution is the basis for our high signal-to-noise ratio and thus our good sensitivity. 


\section{FUTURE WORK}

We will continue to study the performance of the existing prototype tracker as part of our ongoing work. We will also pursue development of a larger prototype tracker with orthogonal layers of scintillating fibers and orthogonal electro-optics. This larger prototype will be more representative of a flight instrument and permit us to address the engineering issues associated with construction, assembly and operation of a large orthogonal-layer tracker. We will be able to develop algorithms for track identification and reconstruction in three dimensions, perform calibrations at higher energies with neutrons protons and gammas and to continue to develop and validate detector response models.

\section{CONCLUSIONS}

The SONTRAC laboratory prototype has demonstrated the important features of the detection technique not addressed in earlier work. This helps to determine the engineering parameters important to the SONTRAC application (scintillating fiber pitch, light yields, gains, photocathode and phosphor selection, gating delays and intervals). It is limited to tracking in two dimensions. Self-triggered images of tracks of $\sim 20 \mathrm{MeV}$ protons, recoil protons from 14 to $\sim 65$ $\mathrm{MeV}$ neutrons and minimum ionizing tracks of cosmic ray muons are clearly resolved. The track images and associated pulse height information provide good resolution measurements of both the direction and energy of the incident radiation. An extension to 3-dimensional tracking promises to provide unprecedented measurement capabilities for studies in a variety of fields.

\section{ACKNOWLEDGMENTS}

We wish to thank Professor Patrick Papin and David Smith at San Diego State University for their assistance with the $14 \mathrm{MeV}$ neutron calibration. We also wish to thank Dr. Carlos Castaneda and his Crocker Laboratory team at the University of California at Davis for their assistance with the higher energy neutron and proton calibrations. We also thank Mr. Tim O'Gorman for simulations and Drs. Glenn Frye and Tom Jenkins for their support.

This work is supported under NASA's Space Physics
Supporting Research and Technology program.

\section{REFERENCES}

[1] D. Herzo, et al, Nucl. Instr. Methods, vol. 123, p 583, 1975.

[2] G.Kanbach, et al., J. Geophys. Res., vol. 79, p 5159, 1974.

[3] J. A. Lockwood, et al., J. Geophys. Res., vol. 81, p 6211, 1976.

[4] J. M. Ryan, et al., in Data Analysis in Astronomy IV, p 261, Plenum Press, NY, 1992.

[5] M. J. Hagyard, et al., Advances in Space Research, vol. 4 (1984): 71.

[6] H. Zirin, Astrophysics of the Sun, New York: Cambridge Univ. Press, 1988

[7] H. Hudson and J. Ryan. Annual Review Astronomy and Astrophysics, vol. 33 (1995): 239-82.

[8] W.T. Vestrand and J.A. Miller. "Particle Acceleration During Solar Flares. The Many Faces of the Sun": The Scientific Results of the Solar Maximum Mission, ed. B. Haisch et al. New York: Springer Verlag, 1994.

[9] Walt, Martin, Introduction to Geomagnetically Trapped Radiation, Cambridge University Press, 1994

[10] A. M. Preszler, et al., J. Geophys. Res., vol. 81, 4715-4722, 1976.

[11] T. Armstrong, et al., 1973, J. Geophys. Res., vol. 78, 2715.

[12] M. Merker, 1975, Proc, ICRC, vol. 4, 1393.

[13] J. F. Ziegler, et al., 1996, IBM Journal of Research and Development, vol. 40, No. 1,3 .

[14] T. O'Gorman, et al., 1996, IBM Journal of Research and Development, vol. 40 , No. 1, 41 .

[15] J. M. Ryan and R. Saxena, "Ground Level Neutron Measurements from 10-170 MeV", Proc. Amer. Nucl. Soc. Topical Meetings, Radiation Protection and Shielding, vol. 1, 219-226 (1996).

[16] J. L. Romero, et al., "Patient positioning for protontherapy using a proton range telescope", Nuclear Instruments and Methods in Physics Research A, vol. 356 (1995),558-565

[17] G. M. Frye, et al., 19th ICRC, vol. 5, 498, 1985

[18] G. M. Frye, et al., 20th ICRC, vol. 4, 392, 1987

[19] Pendleton et al., Workshop on Scint. Fiber Devel., Fermilab, 1093, 1988

[20] R.E. Ansorge, et al., Nuclear Instruments and Methods in Physics Research A, vol. 265 (1988), 33-49

[21] J. M. Ryan, J. Baltgalvis, D. Holslin, J. R. Macri, M. L. McConnell, A. Polichar, C. B. Wunderer, "A prototype for SONTRAC, a scintillating plastic fiber detector for solar neutron spectroscopy", SPIE proceedings series. vol. 3114 (1997 San Diego), p 514 - 525 